\title{
Effect of edible coatings for enhancing shelf-life and quality in Ber (Zizyphus mauritiana Lamk.) fruits
}

\author{
Piyali Dutta", Koyel Dey ${ }^{*}$, Arkendu Ghosh", Nilesh Bhowmick, Arunava Ghosh ${ }^{* *}$ \\ Department of Pomology and Post-Harvest Technology, Uttar Banga Krishi Viswavidyalaya, Pundibari, Cooch \\ Behar - 736165 (WB), INDIA \\ ** Discipline of Agricultural Statistics, Uttar Banga Krishi Viswavidyalaya, Pundibari, Cooch Behar-736165 (WB), INDIA \\ *Present Address: Department of Fruits and Orchard Management,Bidhan Chandra Krishi Viswavidyalaya, \\ Mohanpur, Nadia - 741252 (WB), INDIA \\ *Corresponding author. E.mail- mithi.rani11@gmail.com
}

Received: December 16, 2015; Revised received: May 22, 2016; Accepted: July 31, 2016

\begin{abstract}
An experiment was conducted to study the effect of post-harvest application of different edible coatings like Chitosan $(0.5 \%, 1.0 \%, 2.0 \%)$, Guar gum $(1 \%, 1.5 \%, 2 \%)$, Gum tragacanth $(1.0 \%, 1.5 \%, 2.0 \%)$ on shelf life and quality of $\mathrm{cv}$. BAU ber. Fruits of uniform size were harvested at physiological maturity and treated with various edible coatings. Observations were recorded at intervals of 4 days from storage on physiological loss in weight, fruit length, breadth, colour, TSS, total sugar, reducing sugar,acidity, and ascorbic acid. The results revealed that coating of fruits resulted in reduced loss in fruit weight and higher level of ascorbic acid content, TSS, acidity, total sugar, reducing sugar as compared to the fruits under control. The most effective coating was Guar gum (2\%) that extended the shelf life of ber up to 16 days. Fruits under control had a shelf life of only10 days.
\end{abstract}

Key wards: Ber, Chitosan, Guar gum, Gum tragacanth, Shelf life

\section{INTRODUCTION}

Ber (Ziziphus mauritiana Lam) is an economically important tropical fruit tree belongs to the family Rhamnaceae. Fruit is highly perishable and has poor shelf life (hardly 2-4 days) at ambient condition (Meena et al. 2009).It is highly nutritious, rich in ascorbic acid, contains fairly good amount of vitamin $\mathrm{A}$ and $\mathrm{B}$, minerals like calcium, phosphorus, iron and has high calorific value and ascorbic acid content (Jawanda et al. 1978).Cultivation of ber is popular in present days. With the introduction of ber cv. BAU Ber in the sub-Himalayan Terai region of West Bengal, the area and production of ber has been increased many folds. Due to the surplus of fruits in the local markets during peak season, a substantial quantity goes to waste, resulting in heavy postharvest losses.Edible coatings have long been known to protect perishable food products from deterioration (Debeaufort et al.1998).The purpose is to extend the shelf life of produce and to provide a barrier against hazards. It may retard moisture migration and the loss of volatile compounds, reduce the respiration rate and delay changes in textural properties (Del-Valle et al. 2005). Edible coatings are used to create a modified atmosphere and to reduce weight loss during transport and storage (Baldwin, 1994). In fact, the barrier characteristics to gas exchange for films and coatings are the subjects of much recent interest (Tripathi, 2004). The objective of this study was to examine the effects of the treatment with chitosan, guar gum, gum tragacanth solution on the shelf life of the ber fruit at ambient temperature.

\section{MATERIALS AND METHODS}

Source of fruits and coating materials: To conduct the experiment, fully mature but green ber fruits were collected from farmer's field and immediately brought to the laboratory of the Department of Pomology and Post-harvest Technology, Faculty of Horticulture, at Uttar Banga Krishi Viswavidyalaya, Pundibari, Coochbehar, during the year 2013, for storage after necessary treatments. Uniform sized, defect-free fruits were selected. The fruits after washing in running tap water dried in the shade for few minutes. A set of 90 fruits with 30 fruits per replication were taken each of the following treatments.

Chitosan (purchased from HIMEDIA, Mumbai, India) solutions was done according to the method of Jiang and $\mathrm{Li}$ (2001). To prepare $500 \mathrm{ml}$ of $0.5 \%, 1.0 \%$ and $2 \%(\mathrm{w} / \mathrm{v})$ chitosan solution, accurate weight of $2.5 \mathrm{~g}$, $5.0 \mathrm{~g}$ and $10 \mathrm{~g}$ of chitosan were dispersed in $50 \mathrm{ml}$ of glacial acetic acid, respectively. The $\mathrm{pH}$ of the solution was adjusted to $\mathrm{pH} 6.0$ with $1 \mathrm{M} \mathrm{NaOH}$ and the solutions were made up to $500 \mathrm{ml}$. Guar gum (purchased from HIMEDIA, Mumbai, India) coating solution was 
prepared on the percentage of weight basis with distilled water. $1 \mathrm{gm}, 1.5 \mathrm{gm}$ and $2 \mathrm{gm}$ guar gum powder was mixed with $100 \mathrm{ml}$ of water for the preparation of $1 \%, 1.5 \%$ and $2 \%$ solutions, respectively. Solutions were heated in oven, cooled in air followed by Wijewardane et al. 2013. Gum tragacanth powder (purchased from HIMEDIA,(Mumbai, India) was used in ratio of 10 to $100 \mathrm{ml}(\mathrm{w} / \mathrm{w})$ and was mixed in water ( $\mathrm{pH}$ was 1.70 ), stirred vigorously with a magnetic stirrer on a hotplate for $40 \mathrm{~min}$ and were kept in the refrigerator for $24 \mathrm{hrs}$ (Mohebbi et al. 2012) for using as coating of ber fruit. Fruit samples were analysed for physico-chemical properties at an interval of 3 days after treatments. The percentage of weight loss was calculated based on initial weight and weight at subsequent intervals. The length and breadth (millimeter scale) of ber fruits were measured as an index for shrinkage for each parameter was performed using Proc Glm of Statistical Analysis System (SAS) software (version9.3). Mean separation for different treatment under different parameter were performed using Least Significant Difference(LSD) test $(\mathrm{P} \leq 0.05)$. Normality of residuals under the assumption of ANOVA was tested using Kolmogrov-Smirnov procedure using Proc-Univariate procedure of SAS, (version 9.3). Data transformation was done following the method of Gomez and Gomez (1983) and it was measured by digital verniercallipers at zero time of storage (beginning) and 3 days interval during the storage period. The fruit colour was recorded with the help of Royal Horticulture Society mini colour chart. Total soluble solids (TSS), total sugar and reducing sugar were estimated by the method described by Mazumdar and Majumder, 2003. The acidity and ascorbic acid were estimated by the method described by Rangana (1977).

Treatments: 1.Chitosan (0.5\%), 2.Chitosan (1\%), 3.Chitosan (2\%), 4.Guar gum (1\%), 5.Guar gum (1.5\%), 6.Guar gum(2\%), 7.Gum tragacanth(1\%), 8.Gum tragacanth(1.5\%), 9.Gum tragacanth(2\%), 10.Control(Untreated).

\section{RESULTS AND DISCUSSION}

Physiological loss in weight: On 4 days after treatment of ber fruits, the Physiological loss in weight was found lowest $(7.37 \%$ ) in fruits treated with guar gum $2 \%$ where as it was maximum $(25.71 \%)$ in the fruits treated with chitosan $2 \%$. However, on 16 days after treatment, the Physiological loss in weight was found lowest $(8.01 \%)$ in fruits treated with guar gum $2 \%$ where as it was maximum $(26.41 \%)$ in the fruits treated with guar gum $1.5 \%$. The reduction in weight loss was probably due to the effects of these coatings as a semi permeable barrier against oxygen, carbon dioxide, moisture and solute movement, thereby reducing respiration, water loss and oxidation reaction rates (Baldwin et al. 1999).

Fruit length and breadth: On 4 days after treatment, the fruit length was found highest $(19.3 \mathrm{~mm})$ in fruits treated with guar gum $2 \%$ where as it was minimum $(13.5 \mathrm{~mm})$ in the fruits treated with guar gum $1 \%$. However, on 16 days after treatment, the fruit length was found highest $(9.41 \mathrm{~mm})$ in fruits treated with guar gum $2 \%$ where as it was minimum $(5.15 \mathrm{~mm})$ in the fruits treated with guar gum 1.5\%.On 4 days after treatment of BAU ber fruits, the fruit breadth was found highest $(15.41 \mathrm{~mm})$ in fruits treated with guar gum $2 \%$ where as it was minimum $(10.46 \mathrm{~mm})$ in the fruits under control. However, on 16 days after treatment, the fruit breadth was found highest $(7.56 \mathrm{~mm})$ in fruits treated with guar gum $2 \%$ where as it was minimum $(4.31 \mathrm{~mm})$ in the fruits under control. It might be due to the anti-senescent action of coatings which had an inhibitory effect on ethylene biosynthesis and retard the activity of enzymes responsible for ripening, cell degradation was prevented which in turn facilitated reduced moisture loss and lesser respiratory gas exchange, hence delay in senescence and lower the shrinkage percentage (Sudha et al. 2007).

TSS (Total soluble solid): Observation during storage of fruits revealed that the TSS content was decreased up to the storage period progressed. On 4 days after treatment, the TSS content was found highest $\left(12.27^{\circ}\right.$ brix $)$ in fruits treated with guar gum $2 \%$ where as it was lowest $\left(7.27^{0}\right.$ brix $)$ in the fruits under control. However, on 16 days after treatment, TSS content was found maximum $\left(6.67^{\circ}\right.$ brix $)$ in fruits treated with guar gum $-2 \%$ where as it was minimum $\left(3.6^{\circ}\right.$ brix $)$ in the fruits under control. The increase in TSS and sugar content may be due to the hydrolysis of insoluble polysaccharides into simple sugars. When conversion is lower than the utilization, a decrease of TSS can be seen (Gupta,1987). Rate of increase in TSS under coating treatment may be due to delaying of ripening.

Total sugar: Observation during storage of fruits revealed that the total sugar content was decreased up to the storage period progressed. On 4 days after treatment, the total sugar content was found highest $(10.23 \%)$ in fruits treated with guar gum $2 \%$, chitosan $2 \%$, and gum tragacanth $1.5 \%$ where as it was lowest $(6.2 \%)$ in the fruits under control. However, on 16 days after treatment, total sugar content was found maximum $(5.17 \%)$ in fruits treated with guar gum $2 \%$ where as it was minimum $(3.2 \%)$ in the fruits under control. The change of sugar content is occurred due to utilization of sugar as a respiratory substrate (Nandane and Jain 2011).

Reducing sugar: It is revealed that the reducing sugar content of ber fruits was decreased up to the storage period progressed. On 4 days after treatment of bauber fruit, the reducing sugar content was found highest $(4.66 \%)$ in fruits treated with guar gum $2 \%$ where as it was lowest $(3.3 \%)$ in the fruits under control. However, on 16 days after treatment, total sugar content was found maximum $(1.74 \%)$ in fruits treated with guar gum $2 \%$ 
Piyali Dutta et al. / J. Appl. \& Nat. Sci. 8 (3): 1421 - 1426 (2016)

Table. 1. Effect of some post-harvest treatments on Physiological loss in weight (\%).

\begin{tabular}{llllll}
\hline Treatments & \multicolumn{5}{c}{ Days after storage } \\
\cline { 2 - 6 } & $\mathbf{4}$ & $\mathbf{8}$ & $\mathbf{1 2}$ & $\mathbf{1 6}$ & Cumulative \\
\hline Chitosan(0.5\%) & $8.25 \mathrm{~b}$ & $7.77 \mathrm{a}$ & $7.69 \mathrm{c}$ & $6.47 \mathrm{c}$ & $22.88 \mathrm{e}$ \\
Chitosan(1\%) & $11.57 \mathrm{ab}$ & $8.99 \mathrm{a}$ & $9.17 \mathrm{c}$ & $17.97 \mathrm{abc}$ & $39.82 \mathrm{~d}$ \\
Chitosan(2\%) & $25.71 \mathrm{a}$ & $9.41 \mathrm{a}$ & $11.06 \mathrm{bc}$ & $23.83 \mathrm{ab}$ & $55.43 \mathrm{a}$ \\
Guar gum(1\%) & $10.83 \mathrm{ab}$ & $12.36 \mathrm{a}$ & $12.76 \mathrm{abc}$ & $15.25 \mathrm{abc}$ & $42.39 \mathrm{~cd}$ \\
Guar gum(1.5\%) & $20.4 \mathrm{ab}$ & $12.66 \mathrm{a}$ & $15.81 \mathrm{abc}$ & $26.41 \mathrm{a}$ & $57.08 \mathrm{a}$ \\
Guar gum(2\%) & $7.37 \mathrm{~b}$ & $8.22 \mathrm{a}$ & $7.92 \mathrm{c}$ & $8.01 \mathrm{bc}$ & $25.92 \mathrm{e}$ \\
Gum tragacanth(1\%) & $13.63 \mathrm{ab}$ & $13.44 \mathrm{a}$ & $18.66 \mathrm{ab}$ & $19.48 \mathrm{abc}$ & $51.78 \mathrm{ab}$ \\
Gum tragacanth(1.5\%) & $18.56 \mathrm{ab}$ & $9.51 \mathrm{a}$ & $9.54 \mathrm{bc}$ & $24.21 \mathrm{ab}$ & $49.61 \mathrm{abc}$ \\
Gum tragacanth(2\%) & $19.31 \mathrm{ab}$ & $9.2 \mathrm{a}$ & $13.96 \mathrm{abc}$ & $9.3 \mathrm{bc}$ & $43.3 \mathrm{bcd}$ \\
Control(Untreated) & $11.41 \mathrm{ab}$ & $13.43 \mathrm{a}$ & $20.89 \mathrm{a}$ & $20.68 \mathrm{a}$ & $52.29 \mathrm{a}$ \\
SEM( \pm ) & 79.53 & 30.89 & 29.02 & 95.44 & 26.42 \\
LSD(P $\leq 0.05)$ & 15.18 & 9.46 & 9.17 & 16.63 & 8.75 \\
\hline$* *$ Means with the same letter are not significantly different & & &
\end{tabular}

Table. 2.Effect of some post-harvest treatments on length ( $\mathrm{mm}$.) of fruits

\begin{tabular}{lllll}
\hline Treatments & Days after storage & & $\mathbf{1 6}$ \\
\cline { 2 - 4 } & $\mathbf{4}$ & $\mathbf{8}$ & $\mathbf{1 2}$ & $7.21 \mathrm{abc}$ \\
\hline Chitosan(0.5\%) & $17.94 \mathrm{ab}$ & $15.13 \mathrm{ab}$ & $12.05 \mathrm{a}$ & $5.38 \mathrm{bc}$ \\
Chitosan(1\%) & $18.5 \mathrm{ab}$ & $15.84 \mathrm{a}$ & $11.88 \mathrm{a}$ & $7.03 \mathrm{abc}$ \\
Chitosan(2\%) & $17.44 \mathrm{ab}$ & $15.18 \mathrm{ab}$ & $12.77 \mathrm{a}$ & $6.84 \mathrm{abc}$ \\
Guar gum(1\%) & $13.5 \mathrm{~b}$ & $11.78 \mathrm{bc}$ & $10.5 \mathrm{ab}$ & $5.15 \mathrm{c}$ \\
Guar gum(1.5\%) & $15.42 \mathrm{ab}$ & $11.77 \mathrm{bc}$ & $7.64 \mathrm{bc}$ & $9.41 \mathrm{a}$ \\
Guar gum(2\%) & $19.3 \mathrm{ab}$ & $16.07 \mathrm{a}$ & $13.03 \mathrm{a}$ & $9.13 \mathrm{ab}$ \\
Gum tragacanth(1\%) & $17.58 \mathrm{ab}$ & $15.38 \mathrm{ab}$ & $11.26 \mathrm{a}$ & $6.32 \mathrm{abc}$ \\
Gum tragacanth(1.5\%) & $13.78 \mathrm{~b}$ & $10.68 \mathrm{c}$ & $6.94 \mathrm{c}$ & $7.84 \mathrm{abc}$ \\
Gum tragacanth(2\%) & $17.8 \mathrm{a}$ & $15.54 \mathrm{a}$ & $11.75 \mathrm{a}$ & $7.78 \mathrm{ab}$ \\
Control(Untreated) & $18.75 \mathrm{a}$ & $14.54 \mathrm{ab}$ & $11.57 \mathrm{a}$ & 4.91 \\
SEM( \pm () & 8.93 & 4.73 & 4.05 & 3.80 \\
LSD(P $\leq 0.05)$ & 5.13 & 3.73 & 3.45 & \\
\hline
\end{tabular}

**Mean with the same letter are not significantaly diffrent

Table. 3.Effect of some post-harvest treatments on fruit breadth(mm.)

\begin{tabular}{lcccc}
\hline \multicolumn{1}{c}{ Treatments } & \multicolumn{4}{c}{ Days after treatments } \\
\cline { 2 - 5 } & $\mathbf{4}$ & $\mathbf{8}$ & $\mathbf{1 2}$ & $\mathbf{1 6}$ \\
\hline Chitosan(0.5\%) & $13.51 \mathrm{abc}$ & $11.99 \mathrm{ab}$ & $6.25 \mathrm{a}$ & $5.19 \mathrm{a}$ \\
Chitosan(1\%) & $12.79 \mathrm{abc}$ & $11.07 \mathrm{ab}$ & $9.63 \mathrm{a}$ & $5.25 \mathrm{~b}$ \\
Chitosan(2\%) & $11.94 \mathrm{abc}$ & $9.21 \mathrm{ab}$ & $6.58 \mathrm{ab}$ & $4.75 \mathrm{ab}$ \\
Guar gum(1\%) & $12.01 \mathrm{abc}$ & $9.86 \mathrm{ab}$ & $7.46 \mathrm{ab}$ & $5.63 \mathrm{ab}$ \\
Guar gum(1.5\%) & $11.26 \mathrm{bc}$ & $9.13 \mathrm{ab}$ & $6.97 \mathrm{ab}$ & $4.99 \mathrm{ab}$ \\
Guar gum(2\%) & $15.41 \mathrm{c}$ & $12.68 \mathrm{a}$ & $9.71 \mathrm{~b}$ & $7.56 \mathrm{ab}$ \\
Gum tragacanth(1\%) & $12.39 \mathrm{abc}$ & $9.09 \mathrm{ab}$ & $7.7 \mathrm{ab}$ & $5.78 \mathrm{ab}$ \\
Gum tragacanth(1.5\%) & $14.91 \mathrm{ab}$ & $11.63 \mathrm{ab}$ & $9.49 \mathrm{a}$ & $6.04 \mathrm{ab}$ \\
Gum tragacanth(2\%) & $12.1 \mathrm{abc}$ & $9.87 \mathrm{ab}$ & $7.7 \mathrm{ab}$ & $4.91 \mathrm{ab}$ \\
Control(Untreated) & $10.46 \mathrm{a}$ & $8.19 \mathrm{a}$ & $8.65 \mathrm{ab}$ & $4.31 \mathrm{ab}$ \\
SEM( \pm LSD & 5.74 & 5.26 & 3.62 & 2.84 \\
LSD $\leq 0.05)$ & 4.08 & 3.91 & 3.24 & 2.87 \\
\hline
\end{tabular}

***Means with the same letter are not significantly different

where as it was minimum $(1 \%)$ in the fruits under control.The change of reducing sugar content is occurred due to utilization of sugar as a respiratory substrate (Nandane and Jain 2011).

Ascorbic acid: Observation during storage of ber fruits revealed that the ascorbic acid content (Table 7) was decreased in all the treatments as the storage period advanced. On 4 days after treatment, the ascorbic acid content was found maximum $(90.98 \mathrm{mg})$ with guar gum $2 \%$, where as it was minimum $(89 \mathrm{mg})$ in the fruits under control. However, on 16 days after treatment, the ascorbic acid content was found highest $(78.73 \mathrm{mg}$ ) in fruits treated with guar gum $2 \%$ where as it was lowest (77.03mg) under control. Bhowmick et al. (2015) reported that guar gum not only extends the shelf life but also preserves the ascorbic acid content which is associated with antioxidant capacity during storage and also suggests that guar gum is promising as an edible coating Titrable acidity: It was revealed from this experiment that the titrable acid content (Table 8) was decreased in 
Table . 4. Effect of some post-harvest treatments on TSS \% .

\begin{tabular}{|c|c|c|c|c|}
\hline \multirow[t]{2}{*}{ Treatments } & \multicolumn{4}{|c|}{ Days after storage } \\
\hline & 4 & 8 & 12 & 16 \\
\hline Chitosan $(0.5 \%)$ & $8.2 \mathrm{f}$ & $5.8 \mathrm{e}$ & $5.03 \mathrm{ef}$ & $4.43 \mathrm{de}$ \\
\hline Chitosan(1\%) & $10.13 c$ & $9.1 \mathrm{~b}$ & $7.8 \mathrm{~b}$ & $6.47 \mathrm{ab}$ \\
\hline Chitosan(2\%) & $11.43 b$ & $9.27 \mathrm{~b}$ & $5.13 \mathrm{a}$ & $4.3 \mathrm{a}$ \\
\hline Guar gum(1\%) & $8.97 \mathrm{e}$ & $8.23 \mathrm{c}$ & $7.37 \mathrm{c}$ & $6.57 \mathrm{a}$ \\
\hline Guar gum(1.5\%) & $9.43 \mathrm{~d}$ & $8.33 \mathrm{c}$ & $6.2 \mathrm{~d}$ & $5.53 \mathrm{bc}$ \\
\hline Guar gum $(2 \%)$ & $12.27 \mathrm{a}$ & $10.23 b$ & $8.07 \mathrm{ef}$ & $6.67 \mathrm{de}$ \\
\hline Gum tragacanth(1\%) & $10.3 \mathrm{c}$ & $8.1 \mathrm{c}$ & $5.17 \mathrm{ef}$ & $3.97 \mathrm{de}$ \\
\hline Gum tragacanth $(1.5 \%)$ & $11.2 \mathrm{~b}$ & $9.23 \mathrm{a}$ & $5 f$ & $4.53 \mathrm{de}$ \\
\hline Gum tragacanth $(2 \%)$ & $10.03 \mathrm{c}$ & $7.23 \mathrm{~d}$ & $5.27 \mathrm{e}$ & $4.6 \mathrm{~cd}$ \\
\hline Control(Untreated) & $7.27 \mathrm{~g}$ & $5.77 \mathrm{e}$ & $4.27 \mathrm{~g}$ & $3.6 \mathrm{e}$ \\
\hline $\operatorname{SEM}( \pm)$ & 0.04 & 0.02 & 0.02 & 0.32 \\
\hline $\operatorname{LSD}(\mathrm{P} \leq 0.05)$ & 0.33 & 0.24 & 0.26 & 0.96 \\
\hline
\end{tabular}

**Means with the same letter are not significantly different

Table. 5. Effect of some post-harvest treatments on total sugar \% .

\begin{tabular}{|c|c|c|c|c|}
\hline \multirow[t]{2}{*}{ Treatments } & \multicolumn{4}{|c|}{ Days after storage } \\
\hline & 4 & 8 & 12 & 16 \\
\hline Chitosan $(0.5 \%)$ & $7.23 \mathrm{~d}$ & $4.43 \mathrm{e}$ & $4.03 \mathrm{de}$ & $3.55 \mathrm{c}$ \\
\hline Chitosan $(1 \%)$ & $9.1 b$ & $8 b$ & $6.47 \mathrm{~b}$ & $5.13 \mathrm{a}$ \\
\hline Chitosan(2\%) & $10.23 \mathrm{a}$ & $8.07 \mathrm{~b}$ & $4.27 \mathrm{a}$ & $3.94 b$ \\
\hline Guar gum(1\%) & $8.07 \mathrm{c}$ & $7.37 \mathrm{c}$ & $6.2 \mathrm{~b}$ & $3.32 \mathrm{a}$ \\
\hline Guar gum( $(1.5 \%)$ & $8.07 \mathrm{c}$ & $7.37 \mathrm{~b}$ & $6.2 \mathrm{c}$ & $3.32 b$ \\
\hline Guar gum $(2 \%)$ & $10.23 \mathrm{a}$ & $9.27 \mathrm{~b}$ & $7.37 \mathrm{de}$ & $5.17 \mathrm{~cd}$ \\
\hline Gum tragacanth $(1 \%)$ & $8.23 c$ & $7.27 \mathrm{c}$ & $3.97 \mathrm{de}$ & $3.43 d$ \\
\hline Gum tragacanth $(1.5 \%)$ & $10.23 \mathrm{a}$ & $8.07 \mathrm{a}$ & $4.53 \mathrm{~cd}$ & $3.28 \mathrm{~cd}$ \\
\hline Gum tragacanth(2\%) & $9.23 b$ & $8.07 \mathrm{~b}$ & $3.6 \mathrm{e}$ & $3.09 \mathrm{~cd}$ \\
\hline Control(Untreated) & $6.2 \mathrm{e}$ & $6.37 \mathrm{~d}$ & $4.6 \mathrm{~cd}$ & $3.2 \mathrm{~cd}$ \\
\hline $\operatorname{SEM}( \pm)$ & 0.02 & 0.03 & 0.18 & 0.05 \\
\hline $\operatorname{LSD}(\mathrm{P} \leq 0.05)$ & 0.27 & 0.30 & 0.72 & 0.04 \\
\hline
\end{tabular}

Table. 6. Effect of some post-harvest treatments on reducing sugar \% of fruits.

\begin{tabular}{lcccc}
\hline \multicolumn{1}{c}{ Treatments } & \multicolumn{4}{c}{ Days after treatment } \\
\cline { 2 - 5 } & $\mathbf{4}$ & $\mathbf{8}$ & $\mathbf{1 2}$ & $\mathbf{1 6}$ \\
\hline Chitosan(0.5\%) & $3.47 \mathrm{ef}$ & $2.61 \mathrm{c}$ & $2.33 \mathrm{c}$ & $1.55 \mathrm{a}$ \\
Chitosan(1\%) & $4.42 \mathrm{a}$ & $3.88 \mathrm{ab}$ & $2.85 \mathrm{~b}$ & $1.62 \mathrm{a}$ \\
Chitosan(2\%) & $3.97 \mathrm{bcd}$ & $3.76 \mathrm{~b}$ & $2.31 \mathrm{a}$ & $1.7 \mathrm{a}$ \\
Guar gum(1\%) & $3.41 \mathrm{f}$ & $3.72 \mathrm{~b}$ & $2.76 \mathrm{~b}$ & $1 \mathrm{a}$ \\
Guar gum(1.5\%) & $3.37 \mathrm{f}$ & $3.64 \mathrm{~b}$ & $2.13 \mathrm{~cd}$ & $1.04 \mathrm{~b}$ \\
Guar gum(2\%) & $4.66 \mathrm{ab}$ & $4.17 \mathrm{ab}$ & $3.23 \mathrm{c}$ & $1.74 \mathrm{~b}$ \\
Gum tragacanth(1\%) & $3.56 \mathrm{f}$ & $3.74 \mathrm{~b}$ & $2.04 \mathrm{~d}$ & $1.01 \mathrm{~b}$ \\
Gum tragacanth(1.5\%) & $4.17 \mathrm{abc}$ & $3.86 \mathrm{a}$ & $2.08 \mathrm{~d}$ & $1.02 \mathrm{~b}$ \\
Gum tragacanth(2\%) & $3.91 \mathrm{cde}$ & $3.62 \mathrm{~b}$ & $2.02 \mathrm{~d}$ & $1.1 \mathrm{~b}$ \\
Control(Untreated) & $3.3 \mathrm{def}$ & $2.89 \mathrm{c}$ & $2.13 \mathrm{~cd}$ & $1.02 \mathrm{~b}$ \\
SEM $( \pm)$ & 0.08 & 0.04 & 0.02 & 0.02 \\
LSD(P $\leq 0.05)$ & 0.49 & 0.32 & 0.23 & 0.23 \\
\hline **Means with the same letter are not significantly different & & &
\end{tabular}

**Means with the same letter are not significantly different

all the treatments as the storage period advanced. On 4 days after treatment, the titrable acid content was found maximum $(0.53 \%)$ in fruits treated with guar gum $2 \%$ where as it was minimum $(0.23 \%)$ in the fruits under control. However, on 16 days after treatment, the acid content was found highest $(0.19 \%)$ in fruits treated with guar $2 \%$ where as it was lowest $(0.11)$ in the fruits treated with guar gum $1 \%$.It is also considered that coatings reduce the rate of respiration and may therefore delay the utilization of organic acids (Yamanand Bayoindirli, 2002).

Fruit colour : From Table 9, it is revealed that on 4 days after treatment, the fruit colour was grey brown group in chitosan treated fruits and yellow green group in remaining treated fruits. However, on 16 days after treatment, the fruits colour was yellow green (light) in colour in guar gum treated fruits except the fruits treated with chitosan, gum tragacanth and fruits under 
Piyali Dutta et al. / J. Appl. \& Nat. Sci. 8 (3): 1421 - 1426 (2016)

Table. 7. Effect of some post-harvest treatments on ascorbic acid (mg/100g of fruit pulp) .

\begin{tabular}{lcccc}
\hline \multicolumn{1}{c}{ Treatments } & \multicolumn{4}{c}{ Days after storage } \\
\cline { 2 - 5 } & $\mathbf{4}$ & $\mathbf{8}$ & $\mathbf{1 2}$ & $\mathbf{1 6}$ \\
\hline Chitosan(0.5\%) & $90.2 \mathrm{bcd}$ & $85.5 \mathrm{abc}$ & $82.1 \mathrm{~cd}$ & $78.1 \mathrm{a}$ \\
Chitosan(1\%) & $89.99 \mathrm{~d}$ & $85.1 \mathrm{~cd}$ & $81 \mathrm{e}$ & $77.95 \mathrm{a}$ \\
Chitosan(2\%) & $89.12 \mathrm{e}$ & $85.5 \mathrm{abc}$ & $82.4 \mathrm{abc}$ & $77.4 \mathrm{a}$ \\
Guar gum(1\%) & $90.11 \mathrm{~cd}$ & $85.7 \mathrm{ab}$ & $81.8 \mathrm{~d}$ & $78.2 \mathrm{a}$ \\
Guar gum(1.5\%) & $89.1 \mathrm{e}$ & $84.9 \mathrm{~cd}$ & $82.59 \mathrm{ab}$ & $78.39 \mathrm{a}$ \\
Guar gum(2\%) & $90.98 \mathrm{a}$ & $86.1 \mathrm{a}$ & $82.76 \mathrm{a}$ & $78.73 \mathrm{a}$ \\
Gum tragacanth(1\%) & $90.5 \mathrm{~b}$ & $85.8 \mathrm{ab}$ & $82.19 \mathrm{~cd}$ & $77.99 \mathrm{a}$ \\
Gum tragacanth(1.5\%) & $89 \mathrm{e}$ & $84.7 \mathrm{~d}$ & $82.34 \mathrm{bc}$ & $78.1 \mathrm{a}$ \\
Gum tragacanth(2\%) & $90.45 \mathrm{bc}$ & $85.03 \mathrm{bcd}$ & $81.89 \mathrm{~d}$ & $78.22 \mathrm{a}$ \\
Control(Untreated) & $90.19 \mathrm{bcd}$ & $85 \mathrm{bcd}$ & $82 \mathrm{~cd}$ & $77.03 \mathrm{a}$ \\
SEM( \pm ( $)$ & 0.04 & 0.18 & 0.06 & 1.08 \\
LSD(P $\leq$ 0.05) & 0.35 & 0.72 & 0.41 & 1.77 \\
\hline$* *$ Means with the same letter are not significantly different & & &
\end{tabular}

Table. 8. Effect of some post-harvest treatments on acidity $\%$.

\begin{tabular}{|c|c|c|c|c|}
\hline \multirow[t]{2}{*}{ Treatments } & \multicolumn{4}{|c|}{ Days after storage } \\
\hline & 4 & 8 & 12 & 16 \\
\hline Chitosan $(0.5 \%)$ & $0.47 \mathrm{ab}$ & $0.22 \mathrm{a}$ & $0.16 \mathrm{bc}$ & $0.15 \mathrm{abc}$ \\
\hline Chitosan $(1 \%)$ & $0.37 \mathrm{bc}$ & $0.21 \mathrm{~b}$ & $0.14 \mathrm{c}$ & $0.12 \mathrm{bcd}$ \\
\hline Chitosan(2\%) & $0.23 \mathrm{a}$ & $0.32 \mathrm{a}$ & $0.19 \mathrm{a}$ & $0.15 \mathrm{a}$ \\
\hline Guar $\operatorname{gum}(1 \%)$ & $0.4 \mathrm{~b}$ & $0.21 \mathrm{~b}$ & $0.13 \mathrm{c}$ & $0.11 \mathrm{~d}$ \\
\hline Guar gum $(1.5 \%)$ & $0.28 b c$ & $0.21 b$ & $0.16 \mathrm{bc}$ & $0.12 \mathrm{bcd}$ \\
\hline Guar $\operatorname{gum}(2 \%)$ & $0.53 \mathrm{~d}$ & $0.35 b$ & $0.28 \mathrm{bc}$ & $0.19 \mathrm{abc}$ \\
\hline Gum tragacanth $(1 \%)$ & $0.23 \mathrm{~d}$ & $0.19 b$ & $0.19 b c$ & $0.16 \mathrm{ab}$ \\
\hline Gum tragacanth $(1.5 \%)$ & $0.42 b$ & $0.22 b$ & $0.19 b c$ & $0.14 \mathrm{bcd}$ \\
\hline Gum tragacanth $(2 \%)$ & $0.23 \mathrm{~d}$ & $0.26 \mathrm{ab}$ & $0.21 b$ & $0.16 \mathrm{abc}$ \\
\hline Control(Untreated) & $0.23 \mathrm{~d}$ & $0.26 \mathrm{ab}$ & $0.16 b c$ & $0.12 \mathrm{~cd}$ \\
\hline $\operatorname{SEM}( \pm)$ & 0.003 & 0.003 & 0.001 & 0.0005 \\
\hline $\operatorname{LSD}(\mathrm{P} \leq 0.05)$ & 0.09 & 0.09 & 0.06 & 0.04 \\
\hline
\end{tabular}

Table. 9.Effect of some post-harvest treatments on colour.

\begin{tabular}{lcccc}
\hline \multicolumn{1}{c}{ Treatments } & \multicolumn{4}{c}{ Days after storage } \\
\cline { 2 - 5 } & $\mathbf{4}$ & $\mathbf{8}$ & $\mathbf{1 2}$ & $\mathbf{1 6}$ \\
\hline Chitosan(0.5\%) & GBGN199D & GBG199B & GBGN199C & GBG199B \\
Chitosan(1\%) & GBGN199D & GBGN199B & GBGN199C & GBG199B \\
Chitosan(2\%) & GBGN199D & GBGN199B & GBGN199C & GBG199B \\
Guar gum(1\%) & YGG144B & YGG144D & YGG145A & YGG144B \\
Guar gum(1.5\%) & YGGN144C & YGG144D & YGG145A & YGG144B \\
Guar gum(2\%) & YGG144B & YGG144D & YGG144B & YGG144B \\
Gum tragacanth(1\%) & YGG144B & YGG144D & YGG144B & BG200D \\
Gum tragacanth(1.5\%) & YGGN144C & YGG144D & YGG145A & BG200D \\
Gum tragacanth(2\%) & YGGN144C & YGG144D & YGG144B & BG200D \\
Control(Untreated) & YGG144B & YGG144D & YGG144B & BG200D \\
\hline
\end{tabular}

\section{GBG- GREY BROWN GROUP, YGG- YELLOW GREEN GROUP, BG- BROWN GROUP}

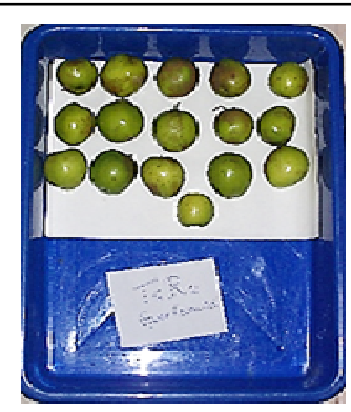

$$
4^{\text {th }} \text { day }
$$

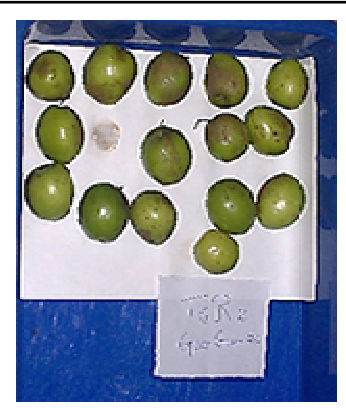

$16^{\text {th }}$ day

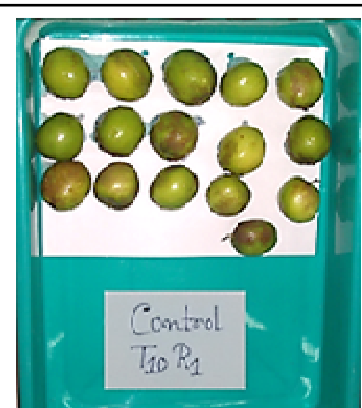

$4^{\text {th }}$ day

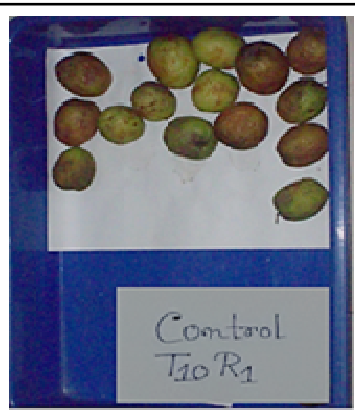

$16^{\text {th }}$ day

Guar gum 2\% treatment

Control fruit 
control which was grey brown group and brown group in colour respectively.

\section{Conclusion}

Ber is a climacteric fruit ripens rapidly after harvest. Edible coating of fruits is a purpose to maintain the nutritional quality and to increase the shelf life. The statistical analysis showed that there was significant variation in all coatings and $2 \%$ guar gum coated ber variety was found significantly superior than other treatments. The results show that the percentage reduction in ascorbic acid, reducing sugars and titratable acidity are less in application of guar gum; higher levels of nutrients are maintained in fruits during storage. The study provides minimizing post-harvest losses by using appropriate edible coating. The extent to which variability in fruit quality at harvest influences shelf life and future research as well as organoleptic tests should be done to determine the acceptance of the stored fruits by consumers.

\section{REFERENCES}

Mazumdar, B. C., and Majumder, K. (2003). Determination of chemical constituents. In: Methods on physiochemical analysis of fruits, Daya publishing House, delhi, pp. 93-139.

Meena, H. R., Kingsly, A. R. P., Jain, R. K. (2009). Effect post-harvest treatments on shelf life of ber fruits. Indian Journal of Horticultur. 66(1): 58-61.

Baldwin, E. A. (1994). Edible coatings for fresh fruits and vegetables: past, present and future. In Edible Coating and Films to Improve Food Quality, pp. 25-64, Technomic Publishing Co., Lancaster, PA.

Baldwin, E. A., Burns, J. K., Kazokas, W., Brecht, J. K., Hagenmaier, R. D., Bender, R. J. and Peris, E. (1999). Effect of two edible coatings with different permeability characteristics on mango (Mangifera indica L.) ripening during storage. Postharvest Biol Technol.17:215-226.

Bhowmick, N., Ghosh, A., Dutta, P. and Dey, K. (2015) .Efficacy of edible coatings on the shelf life of ber (Zizyphus mauritiana Lamk.) fruits at ambient condition. International Journal of Agriculture, Environment and Biotechnology. 8(3): 601-608.

Debeaufort, F. J. A. Quezada-Gallo and Voilley, A. (1998). Edible films and coatings: tomorrow's packagings: a review. Crit. Rev. Food Sci., 38: 299-313.

Del-Valle, V., Hernandez-Munoz, P, Guarda, A. and Galotto, M. J. (2005). Development of a cactus-mucilage edible coating (Opuntiaficus indica) and its application to extend strawberry (Fragaria ananassa) shelf-life. Food Chemistry, 91: 751-756.

Gomez, K. A., Gomez, A. A. (1983). Problem data. Statistical procedures for agricultural research, 2nd edition. Wiley- Inter science Publication (John Wiley and Sons). New York, USA. pp. 275-315.

Gupta, O. P. and Metha, N. (1987). Effect of post-harvest application of fungicides chemicals and pre 100 ling treatment's on the shelf life of Galaber fruits. Hrayana Agriculture University, pp. 561-580.

Jawanda, J. S. and Bal, J. S., (1978), The ber highly paying and rich in food value. Indian Horticulture, 23: 19-21.

Jiang Y., Li Y., (2001). Effects of chitosan coating on postharvest life and quality of longan fruit. Food Chem., 73: $139-143$.

Mohebbi, M., Ansarifar, E., Hasanpour, N., Amiryousefi, M. R. (2012). Suitability of aloe vera and gum tragacanth as edible coatings for extending the shelf life of button mushroom. Food Biotechnology. 5: 3193-3202.

Nandane, A. S., Jain, R. K. (2011). Effect of composite edible coating on physicochemical properties of tomatoes stored at ambient conditions. Indian Journal of Advanced Engineering Technology. 2 (4): 211-17.

Rangana, S. (1977). Ascorbic acid. In: Manual of analysis of fruits and vegetable products. Tata and Mc. Graw Hill Publishing company limited: New Delhi, India, pp. 94101.

Sudha, R., Amutha, R., Muthulaksmi, S., Baby Rani, W., Indira, K., Mareeswari, P. (2007). Influence of pre and post-harvest chemical treatments on physical characteristics of sapota (Achras sapota L.) Var. PKM-1. Research Journal of Agriculture and Biological Sciences, 3(5):450-52.

Tripathi, P. and Dubey. N. K. (2004). Exploitation of natural products as an alternative strategy to control postharvest fungal rotting of fruit and vegetables. Postharvest Biol. Technol. 32(2): 235-245.

Wijewardane, R. M. N. A. (2013). Application of polysaccharide based composite film wax coating for shelf life extension of guava (var. Bangkok Giant). J. of Posthar Tech., 1(1): 16-21.

Yamanand, O. and Bayoindirli, L. (2002). Effects of an edible coating and cold storage on shelf-life and quality of cherries. Lebnsm. Wiss. Und. Technol. 35: 46-150. 